\title{
Germinação de sementes de Bromelia antiacantha em diferentes fotoperíodos
}

\author{
Najlah Patricia Aires Nasser², Rodrigo Ferraz Ramos', Nadine Berwanger Scheeren², Daiane Dalla Nora ${ }^{3}$, \\ Cristiano Bellée, Débora Leitzke Betemps² \\ ' Laboratório de Biologia do Solo, Universidade Federal de Santa Maria (UFSM), Santa Maria, Brasil. \\ Email: rodrigoferrazramos@gmail.com \\ 2 Laboratório de Agroecologia, Universidade Federal da Fronteira Sul (UFFS), Cerro Largo, Brasil. \\ Email: najlah.nasser@hotmail.com, nadinescheeren@gmail.com, debora.betemps@uffs.edu.br \\ 3 Phytus Group, Santa Maria, Brasil. \\ Email: crbelle@gmail.com, daiadallanoraagro@gmail.com
}

\section{Resumo}

O conhecimento do comportamento germinativo de espécies nativas é de significativa importância para a adoção de técnicas que visam sua propagação e conservação. O presente estudo objetivou avaliar o efeito do fotoperíodo na germinação de sementes Bromelia antiacantha Bertol. O delineamento foi inteiramente casualizado, com quatro tratamentos e seis repetições de 25 sementes. Os diferentes fotoperíodos foram $0 \mathrm{~h}, 8 \mathrm{~h}, 12 \mathrm{~h}$ e $16 \mathrm{~h}$ de luz dia ${ }^{-1}$. Avaliou-se o percentual de sementes germinadas $(\mathrm{G})$, índice de velocidade de germinação (IVG), percentual de plântulas normais (PN), anormais (PA) e sementes não germinadas (SNG). A luz não foi um fator limitante na germinação das sementes, contudo, ocorreu aumento progressivo no G, IVG e PN conforme aumento do fotoperíodo, com máxima G (78,0\%) e porcentagem de PN (74,7\%) com I6h luz dia ${ }^{-1}$, e máximo IVG $(19,4)$ com I $2 \mathrm{~h}$ luz dia ${ }^{-1}$. Conclui-se que $B$. antiacantha é uma espécie com sementes fotoblásticas neutras cuja germinação é favorecida na presença de luz.

Palavras-chave: Caraguatá. luz. potencial germinativo.

\section{Abstract}

\section{Germination of Bromelia antiacantha seeds in different photoperiods}

The knowledge of the germinative behavior of native species is important for the adoption of techniques aimed at its propagation and conservation. The present study aimed to evaluate the effect of the photoperiod on the germination of Bromelia antiacantha Bertol seeds. The design was completely randomized, with four treatments and six replicates of 25 seeds. The different photoperiods were $0 \mathrm{~h}, 8 \mathrm{~h}, 12 \mathrm{~h}$ and $16 \mathrm{~h}$ of day ${ }^{-1}$ light. The percentage of germinated seeds (G), germination speed index (IVG), percentage of normal seedlings (PN), abnormal seedlings (AP) and non-germinated seeds (NG) were evaluated. However, there was a progressive increase in G, IVG and PN as the photoperiod increased, with maximum G (78.0\%) and percentage of PN (74.7\%) with I6h light day ${ }^{-1}$, and maximum IVG (I9.4) with I2h day ${ }^{-1}$ light. We conclude that $B$. antiacantha is a species with neutral photoblastic seeds whose germination is favored in the presence of light.

Keywords: Caraguatá. Light. Potential Germination.

\section{Introdução}

A família botânica Bromeliaceae possui aproximadamente 3 I40 espécies distribuídas em 58 gêneros, apresentando distribuição geográfica tipicamente neotropical (GIVNISH et al., 20I I). A maior diversidade dessa família encontra-se na América do Sul, com cerca de 73\% dos gêneros e 40\% das espécies (AOYAMA 
et al., 20I2). Estima-se que mais de 90 espécies das Bromeliaceae são utilizadas no mundo todo para diversos fins, principalmente enquanto plantas ornamentais de grande valor comercial e fonte de alimento, além de apresentarem propriedades medicinais (BERED et al., 2008).

A espécie Bromelia antiacantha Bertol. (Bromeliaceae), conhecida vulgarmente como caraguatá, apresenta valor medicinal e alimentar (WANDERLEY et al., 2007), e compõe as espécies nativas da região Sul do Brasil (FLORARS, 20I2). Esta espécie pode ser propagada vegetativamente (estolão) ou por propagação seminal (LORENZI; MATOS, 2008). Contudo, o uso de sementes como material propagativo é favorecido devido a elevada produção de sementes e a uma alta resistência destas à períodos longos de armazenamento e muitas vezes depende da presença ou ausência luz para iniciar o processo de germinação (DETTKE; MILANEZE-GUTIERRE, 2008).

A luz é um dos diversos fatores que influencia na germinação das sementes, onde aquelas que necessitam luminosidade para germinar são denominadas fotoblásticas positivas; já aquelas que germinam melhor na ausência de luz são fotoblásticas negativas e quando não há interferência luminosa na germinação as sementes são fotoblásticas neutras (NOGUEIRA et al., 20I4). Assim, a germinação das sementes em relação à luz é uma resposta ecofisiológica da espécie, e no caso de espécies selvagens, tem estreita correspondência com o seu posicionamento no estádio sucessional da floresta (SILVA et al., 2002; MAEKAWA et al., 2010).

Em geral, sementes de muitas espécies cultivadas são indiferentes à luz para germinar, entretanto, o estímulo luminoso é bastante variável em espécies selvagens (ROSA; FERREIRA, 1998). No caso das bromélias, muitas requerem a presença de luz para que ocorra a germinação (AOYAMA et al., 2012). Contudo, apesar de a luz ser um fator importante para a família Bromeliaceae, seus efeitos na germinação de sementes de $B$. antiacantha são pouco detalhados na literatura científica. Nesse sentido, o presente trabalho objetivou avaliar o efeito de diferentes fotoperíodos em sementes B. antiacantha.

\section{Material e Métodos}

Frutos maduros de B. antiacantha foram coletados conforme Krumreich et al. (2015) no município de

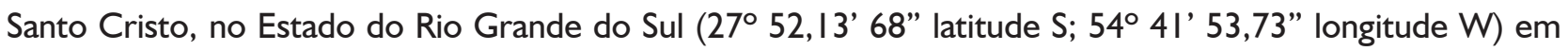
junho do ano de 2017. Os frutos foram conduzidos ao laboratório de Fisiologia Vegetal da Universidade Federal da Fronteira Sul (UFFS), campus Cerro Largo, sendo despolpados e as sementes obtidas foram limpas conforme metodologia de Braga Filho et al. (20l4). Após, foram submetidas à secagem, e armazenadas em saco de papel por um período de um (I) ano em temperatura ambiente com proteção à luz. Na data de realização dos testes, as sementes foram desinfestadas em hipoclorito de sódio $(\mathrm{NaClO})$ a $1 \%$ durante I minuto, agitadas constantemente e enxaguadas em água destilada por 3 vezes.

Os testes de germinação foram realizados em incubadora do tipo Biochemical Oxygen Demand (B.O.D.), com lâmpada tubular fluorescente $20 \mathrm{~W}$ T- $105000 \mathrm{~K}$ branca fria, e em temperatura controlada de $25^{\circ} \mathrm{C}$. Utilizou-se delineamento inteiramente casualizado (DIC), com quatro (4) tratamentos e seis (6) repetições. Em cada unidade experimental acondicionou-se 25 sementes, totalizando 600 sementes em todo o experimento. Os diferentes tratamentos referem-se a diferentes períodos de adição de luz: $0 \mathrm{~h}$ ( $24 \mathrm{~h}$ escuro), $8 \mathrm{~h}, 12 \mathrm{~h}$ e $16 \mathrm{~h}$ de luz ao dia.

As sementes foram semeadas em caixas de acrílico do tipo Gerbox (II cm $\times 1 \mathrm{I} \mathrm{cm} \times 4 \mathrm{~cm}$ ), previamente higienizadas com $\mathrm{NaClO}$ a $1 \%$, em seguida lavadas em água destilada e colocadas para secar. Utilizou-se semeadura sobre papel, colocando sobre duas folhas de papel do tipo germitest umedecidos com $8 \mathrm{ml}$ de água destilada e, acondicionadas em B.O.D nas horas de luz/escuro conforme os tratamentos descritos. As avaliações do percentual germinativo foram realizadas durante período de julho a agosto de 20 I 8 .

Realizou-se a contagem diária de sementes geminadas de acordo com diferentes regimes de adição de luz, sendo consideradas sementes germinadas quando estas apresentaram no mínimo 2 milímetros $(\mathrm{mm})$ de radícula. Posteriormente, foi calculada a porcentagem de germinação de acordo com a fórmula descrita por Carvalho e Carvalho (2009). O índice de velocidade de germinação (IVG) foi calculado conforme descrito por Maguire (1962). A determinação de plântulas normais e anormais foi realizada de acordo os padrões estabelecidos pelas Regras para Análise de Sementes (BRASIL, 2009) e os dados obtidos foram expressos em porcentagem.

Os dados obtidos foram analisados quanto à normalidade pelo teste de Shapiro Wilk e à homocedasticidade pelo teste de Hartley através do Software R Studio (R CORE TEAM, 20I8). Posteriormente, os dados foram submetidos à análise de variância $(p \leq 0,05)$ e em caso de significância estatística as médias foram comparadas pelo teste de Tukey $(p \leq 0,05)$ com o programa estatístico SISVAR (FERREIRA, 20I I). 


\section{Resultados e Discussão}

$\mathrm{Na}$ Figura IA é apresentada a progressão da germinação para cada tratamento durante 26 dias de avaliação. Verifica-se que ocorreu um aumento progressivo no percentual de germinação das sementes conforme aumento do fotoperíodo. Os tratamentos com $0 \mathrm{~h}, 8 \mathrm{~h}, 12 \mathrm{~h}$ e $16 \mathrm{~h}$ de luz dia ${ }^{-1}$ resultaram em $53,3 \%, 61,3 \%$, $76,0 \%$ e $78,0 \%$ de germinação, respectivamente. Os resultados obtidos denotam que a porcentagem de germinação com Oh (ausência de exposições à luz) e $8 \mathrm{~h}$ de luz dia ${ }^{-1}$ não diferem estatisticamente entre si, indicando uma resposta fotoblástica neutra (Tabela I). Contudo, observa-se que os tratamentos com I $2 \mathrm{~h} \mathrm{e}$ $16 \mathrm{~h}$ de luz $\mathrm{dia}^{-1}$ resultaram significativamente nas maiores porcentagens de germinação, diferindo estatisticamente dos tratamentos com $0 \mathrm{~h}$ e $8 \mathrm{~h}$ de luz dia-1.

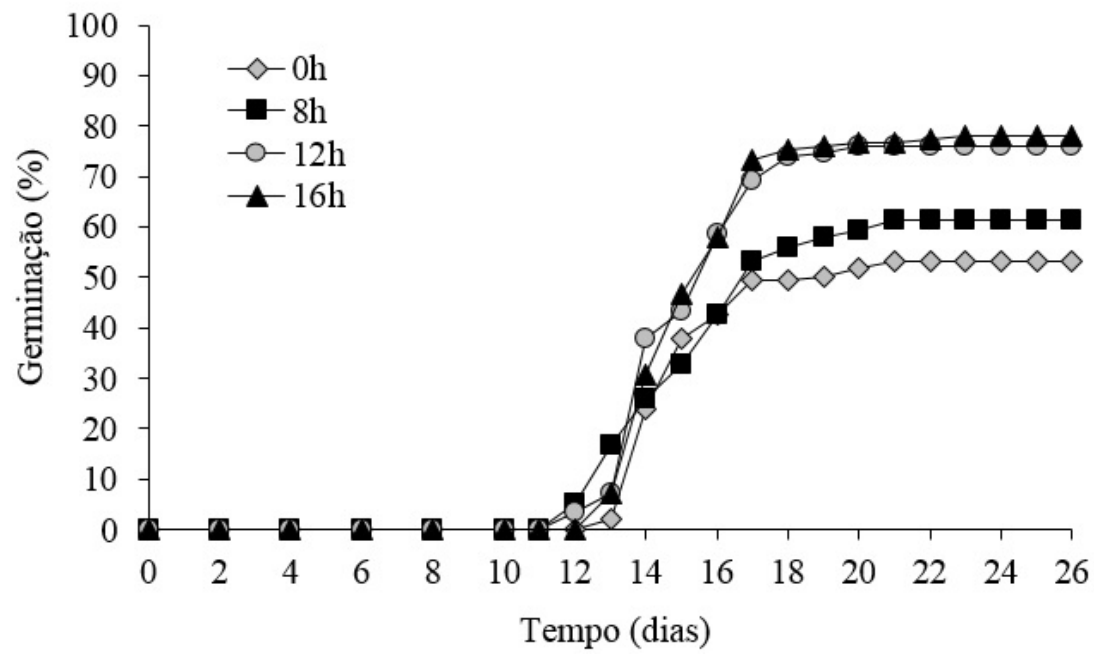

Figura I

Progressão da germinação de sementes de Bromelia antiacantha submetidas a diferentes fotoperíodos durante 26 dias de avaliação

\begin{tabular}{lcc}
\hline Horas de luz dia-1 & G (\%) & IVG \\
\hline Oh & $53,3 \mathrm{~b}^{\prime}$ & $7,9 \mathrm{~b}$ \\
$8 \mathrm{~h}$ & $61,3 \mathrm{~b}$ & $15, \mathrm{I} \mathrm{a}$ \\
$\mathrm{I} 2 \mathrm{~h}$ & $76,0 \mathrm{a}$ & $19,4 \mathrm{a}$ \\
$16 \mathrm{~h}$ & $78,0 \mathrm{a}$ & $11,4 \mathrm{~b}$ \\
\hline C.V. $(\%)$ & 19,6 & 20,5 \\
\hline
\end{tabular}

' Letras diferentes na coluna denotam diferenças significativas entre os tratamentos pelo teste de Tukey $(p \leq 0,05)$.

Diante dos resultados supracitados, observa-se que as sementes de $B$. antiacantha demonstraram-se sensíveis ao estímulo luminoso, indicando que a luz, apesar de não ser um fator limitante, é um fator que influencia positivamente a germinação dessa espécie. De acordo com Rosa e Ferreira (1998) e Dettke e Milaneze-Gutierre (2008) a germinação das sementes de B. antiacantha na ausência de exposições à luz indica que essa espécie é fotoblástica neutra. Resultados semelhantes foram reportado por Silveira et al. (2004) em Marcetia taxifolia (A. St.-Hil.) DC., onde ocorreu germinação das sementes na ausência de luz, contudo, observando-se aumento na germinação com adição de fotoperíodo de 12 horas luz dia ${ }^{-1}$.

Corroborando com os resultados do presente estudo, Rios et al. (2016) observaram que a bromélia Aechmea costantinii (Mez) L. B. Sm. apresenta germinação das sementes independentemente da presença de luz. Contudo, a germinação de diversas espécies de bromélias depende da presença de luz (AOYAMA et al., 2012), como ocorre com as espécies Aechmea nudicaulis (L.) Griesebach e Streptocalyx floribundus (Martius ex Schultes F.) Mez. (PINHEIRO, BORGHETTI, 2003), e Dyckia distachya Hassler, que são consideradas fotoblásticas positivas (WIESBAUER et al., 2007). 
O aumento do fotoperíodo resultou em um acréscimo no índice de velocidade de germinação (IVG), onde os fotoperíodos de $8 \mathrm{~h}$ e $12 \mathrm{~h} \mathrm{luz} \mathrm{dia}^{-1}$ resultaram significativamente nos maiores valores de IVG (Tabela I), diferindo estatisticamente do tratamento com ausência de exposições à luz (Oh de luz $\mathrm{dia}^{-1}$ ) e do tratamento com fotoperíodo de 16 horas de luz dia-1 (Tabela I). Assim, embora tenha se observado um aumento do IVG no fotoperíodo de 16 horas de luz dia ${ }^{-1}$, este não diferiu estatisticamente do tratamento em escuro pleno (Oh luz $\mathrm{dia}^{-1}$ ).

De maneira geral, no ambiente em pleno escuro (fotoperíodo de 0 horas luz $\mathrm{dia}^{-1}$ ) registrou-se o menor IVG, enquanto que a luz demonstrou-se um fator que influencia positivamente $o$ índice de velocidade de germinação de B. antiacantha. Resultados contrários foram reportados por Rosa e Ferreira (1998), onde o processo germinativo dessa espécie foi menor sob luz contínua (8h luz $\mathrm{dia}^{-1}$ ), quando comparado com os resultados dos tratamentos em pleno escuro. A divergência desses resultados pode ser consequência da variabilidade genética dessa espécie, que apresenta uma ampla distribuição geográfica no Brasil, sendo adaptada a condições edafoclimáticas do Cerrado, Mata Mesófila, Mata Atlântica e Mata de Restinga (WANDERLEY et al., 2007). De acordo com Maekawa et al. (2010), a variação da sensibilidade das sementes à luz pode ocorrer entre espécies, bem como, podem ocorrer variações numa única planta.

Para a variável porcentagem de plântulas normais $(\mathrm{PN})$, plântulas anormais (PA) e sementes não germinadas (SNG), o aumento no fotoperíodo resultou em aumento progressivo no percentual de PN e diminuição do percentual de PN e SNG (Tabela 2). Os tratamentos com $16 \mathrm{~h}$ e $12 \mathrm{~h}$ de luz dia-1 resultaram estatisticamente nas maiores porcentagens de PN (Tabela 2). Resultados intermediários foram obtidos no fotoperíodo de $8 \mathrm{~h}$ de luz $\mathrm{dia}^{-1}$, enquanto que o período de escuro contínuo ( 0 hora de luz $\left.\mathrm{dia}^{-1}\right)$ interferiu, expressivamente, sobre o comportamento das plântulas, registrando estatisticamente o menor percentual médio de $\mathrm{PN}$ em concomitante com maior percentual de PA. $\mathrm{O}$ fotoperíodo de 0 hora de luz dia ${ }^{-1}$ resultou na maior porcentagem de SNG, contudo, não diferindo estatisticamente do fotoperíodo de $8 \mathrm{~h}$ luz $\mathrm{dia}^{-1}$.

Ainda, ressalta-se que os fotoperíodos de $0 \mathrm{~h}$ e $8 \mathrm{~h}$ luz $\mathrm{dia}^{-1}$ não apresentaram diferenças estatísticas entre si para as variáveis germinação (Tabela I) e sementes não germinadas (Tabela 2), contudo, diferiram expressivamente quanto ao percentual de plântulas anormais, onde o tratamento com escuro contínuo $(0$ hora de luz $\mathrm{dia}^{-1}$ ) resultou estatisticamente no menor percentual de PN, enquanto que o tratamento com $8 \mathrm{~h}$ luz $\mathrm{dia}^{-1}$ apresentou resultados semelhantes a exposição das sementes aos fotoperíodos de $12 \mathrm{~h}$ e $16 \mathrm{~h}$ de luz $\mathrm{dia}^{-1}$. Esses resultados corroboram com a assertiva de que a luz é um fator que influencia positivamente a germinação das sementes de $B$. antiacantha.

Tabela 2. Percentual de plântulas normais (PN), plântulas anormais (PA) e sementes não germinadas (SNG) de Bromelia antiacantha submetidos a diferentes fotoperíodos.

\begin{tabular}{cccc} 
Horas de luz dia-1 & PN (\%) & PA (\%) & SNG (\%) \\
\hline Oh & $0,0 \mathrm{c}^{\prime}$ & $53,3 \mathrm{a}$ & $46,7 \mathrm{a}$ \\
$8 \mathrm{~h}$ & $58,0 \mathrm{~b}$ & $3,3 \mathrm{~b}$ & $38,7 \mathrm{a}$ \\
$12 \mathrm{~h}$ & $71,3 \mathrm{a}$ & $4,7 \mathrm{~b}$ & $24,0 \mathrm{~b}$ \\
16h & $74,7 \mathrm{a}$ & $3,3 \mathrm{~b}$ & $22,0 \mathrm{~b}$ \\
\hline C.V. (\%) & 23,5 & 28,0 & 29,3 \\
\hline
\end{tabular}

' Letras diferentes na coluna denotam diferenças significativas entre os tratamentos pelo teste de Tukey $(p \leq 0,05)$.

A germinação de sementes em relação à luz é uma resposta ecofisiológica da espécie, e tem estreita correspondência com o posicionamento no estádio sucessional da floresta (MAEKAWA et al., 2010). De acordo com Aud (2008) a dependência da luz para germinação possui relação direta com o tamanho das sementes, ocorrendo uma diminuição no requerimento por luz com o aumento das reservas das sementes. Nesse sentido, o fotoblastismo em sementes pequenas é um mecanismo para que a germinação dessas espécies ocorra próximo da superfície do solo, aproveitando aberturas no dossel vegetativo, e assim, favorecendo o desenvolvimento inicial das plântulas.

Diante do exposto, destaca-se que os resultados obtidos no presente estudo indicam que a espécie $B$. antiacantha apresenta germinação das sementes em uma ampla faixa de exposição a luz, onde apesar desse 
fator não ser limitante para a germinação das sementes dessa espécie, apresenta-se enquanto um fator que favorece a o processo germinativo. Destaca-se que o conhecimento do comportamento germinativo dessa espécie contribui para a adoção de técnicas que visam sua propagação e conservação na natureza. Nesse contexto, consideram-se prioritárias pesquisas que contemplem a família Bromeliaceae, devido ao interesse científico que elas proporcionam face ao rico germoplasma que apresentam, bem como, a crescente utilização como plantas ornamentais (VIZENTIN et al., 20I6).

\section{Conclusão}

A B. antiacantha é uma espécie com sementes fotoblásticas neutras cuja germinação é favorecida na presença de luz.

\section{Contribuição dos autores}

Todos os autores contribuíram substancialmente para o trabalho aqui relatado. N.P.A.N. e N.B.S. conduziram os experimentos em laboratório. R.F.R. analisou e interpretou os dados, revisou e editou o manuscrito. C.B. e D.D.N. revisaram o manuscrito. D.L.B. coordenou o projeto e supervisionou as atividades. Todos os autores leram e aprovaram o manuscrito final.

\section{Referências}

AOYAMA, E. M.; GONTIJO, A. B. P. L.; FARIA, D. V. Propagação em Bromeliaceae: germinação de sementes e cultivo in vitro. Enciclopédia Biosfera, v.8, n. 15, p. 1452-I47I, 2012.

AUD, F. F. Luz, temperatura e fumaça na germinação de espécies pioneiras da Amazônia Central. 2008. 44 f. Dissertação (Mestrado em Biologia Tropical e Recursos Naturais) - Instituto Nacional de Pesquisas da Amazônia Universidade Federal do Amazonas. Manaus, 2008.

BERED, F. et al. Bromélias. A beleza exótica do Novo Mundo. In: BARBIERI, R. L.; STUMPF, E. R. T. Origem e evolução das plantas cultivadas. Brasília, DF: Embrapa Informação Tecnológica, 2008. p. 235-252.

BRAGA FILHO, J. R. et al. Germinação de sementes e emergência de plântulas de araticum oriundos do Cerrado de Goiás. Bioscience Journal, v. 30, n. I, p. 74-8I, 2014.

BRASIL. Ministério da Agricultura e Reforma Agrária. Regras para análise de sementes. Brasília: Mapa/ACS, 2009.

CARVALHO, D. B.; CARVALHO, R. I. N. Qualidade fisiológica de sementes de guanxuma em influência do envelhecimento acelerado e da luz. Acta Scientiarum Agronomy, v. 3I, n. 3, p. 489-494, 2009.

DETTKE, G. A.; MILANEZE-GUTIERRE, M. A. Anatomia vegetativa de Bromelia antiacantha Bertol. (Bromeliaceae, Bromelioideae). Balduinia, v. 13, n. I, p. I-14, 2008.

FERREIRA, D. F. Sisvar: a computer statistical analysis system. Ciência e Agrotecnologia, v. 35, n. 6, p. 1039-1042, 2011 .

GIEHL, E. L. H. Projeto Flora Digital. In: VERDI, M. Bromelia antiacantha Bertol. Porto Alegre: FLORARS, 2012. Disponível em:

http://www.ufrgs.br/fitoecologia/florars/open_sp.php?img=7653. Acessado em: 08 jan. 2019.

GIVNISH, T.J. et al. Phylogeny, adaptive radiation, and historical biogeography in Bromeliaceae: insights from a neight-locus plastid phylogeny. American Journal of Botany, v. 98, n. 5, p. 872-895. 201 I.

KRUMREICH, F.D.; CORRÊA, A.P.A.; SILVA, S.D.S.; ZAMBIAZI, R.C. Composição físico-química e de compostos bioativos em frutos de Bromelia antiacantha Bertol. Revista Brasileira de Fruticultura, v. 37, n. 2, p. 450-456, 2015.

LORENZI, H.; MATOS, F. J. A. (Org.). Plantas medicinais no Brasil: Nativas e Exóticas. 2.ed. Nova Odessa, SP: Instituto Plantarum, 2008. $544 \mathrm{p}$.

MAEKAWA, L.; ALBUQUERQUE, M. C. F.; COELHO, M. F. B. Germinação de sementes de Aristolochia esperanzae O. Kuntze em diferentes temperaturas e condições de luminosidade. Revista Brasileira de Plantas Medicinais, v. 12, n. I, p. 23-30, 2010. 
MAGUIRE, J. D. Speeds of germination-aid selection and evaluation for seedling emergence and vigor. Crop Science, v. 2, n. I, p. 176-177, 1962.

NOGUEIRA, F. C. B.; GALLÃO, M. I.; BEZERRA, A. M. E.; FILHO, S. M. Efeito da temperatura e luz na germinação de sementes de Dalbergia cearensis Ducke. Ciência Florestal, v. 24, n. 4, p. 995-1005, 2014.

PINHEIRO, F.; BORGHETTI, F. Light and temperature requirements for germination of seeds of Aechmea nudicaulis (L.) Griesebach and Streptocalyx floribundus (Martius ex Schultes f.) Mez (Bromeliaceae). Acta Botânica Brasileira, v. I7, n. I, p. 27-35. 2003.

RIOS, P. A. F.; NETO, J. C. A.; FERREIRA, V. M.; NEVES, M. I. R. S. Seed morphometry and germination of Aechmea costantinii (Mez) L. B. Sm. (Bromeliaceae). Revista Caatinga, v. 29, n. I, p. 85-93, 2016.

ROSA, S. G. T.; FERREIRA, A. G. Germinação de sementes de espécies medicinais do Rio Grande do Sul: Bromelia antiacantaha Bert., Cuphea carthagenensis (Jacq.) Macbride e Talinumpatens (Jacq.) Willdenow. Acta Botânica Brasileira, v. 12, n. 3, 1998.

SILVA, L. M. M.; RODRIGUES, T. J. D.; AGUIAR, I. B. Efeito da luz e da temperatura na germinação de sementes de aroeira (Myracrodruon urundeuva Allemão). Revista Árvore, v. 26, n. 6, p. 691-697, 2002.

SILVEIRA, F. A. O.; NEGREIROS, D.; FERNANDES, G. W. Influência da luz e da temperatura na germinação de sementes de Marcetia taxifolia (A. St.-Hil.) DC. (Melastomataceae). Acta Botânica Brasileira, v. I8, n. 4, p. 847-85I, 2004.

THE R FOUNDATION. R: A language and environment for statistical computing. Vienna: R Core Team, 2018.

VIZENTIN, E. T. B. S.; SOUZA, M. D. A.; SILVA, I. V. Morfologia do fruto, semente e desenvolvimento pós-seminal de Aechmea angustifolia Poepp. \& Endl. (Bromeliaceae). Magistra, v. 28, n. I, p. II0-II8, 2016.

WANDERLEY, M. G. L.; SHEPHERD, G. J; MELHEM, T. S; GIULIETTI, A. M. Flora fanerogâmica do Estado de São Paulo. São Paulo: Instituto de Botânica, 2007.

WIESBAUER, M. B.; SCARIOT, E. C.; SASAKI, L. L.; REIS, A. influência da luz e inundação na germinação de Dyckia distachya Hassler, uma bromélia em vias de extinção. Revista Brasileira de Biociências, v. 5, n. I, p. 7I7-7I9, 2007. 